\title{
Stem cells: tools for dental tissues regeneration
}

\author{
SCs: embryonic, adult, characterization and \\ potential application
}

\section{Characterization of stem cells}

The 200 different somatic cell types of the human body have a limited proliferative capacity. They undergo 50-60 population doublings before entering into senescence, a non-reversible growth arrest. Stem cells bypass this replicative barrier and acquire the ability to divide indefinitely. Three types of stem cells have been identified: embryonic stem cells, cord blood/placental stem cells and adult stem cells. Adult human stem cells are capable of maintaining, generating, and replacing terminally differentiated cells. ${ }^{1}$ Mesenchymal stem cells (MSCs) are multipotent cells. They give rise to mesenchymal and non-mesenchymal tissues in vitro and in vivo. It is clear that (Trans) differentiation capabilities are well documented, however their distribution in the living organisms still needs to be elucidated. ${ }^{2}$ Stem cells are characterized by their ability to promote self-renew capacities. They differentiate into multiple cell lineages derived from the three embryonic germ layers: ectoderm, which is believed to give rise to skin and neural lineages, mesoderm (believed to generate blood, bone, muscle, cartilage and fat), and endoderm (contributing to tissues of the respiratory and digestive tracts). Adult stem cells represent a rare population of multipotent cells in the adult body. Under certain circumstances stem cells may transdifferentiate. The transdifferentiation correspond to the conversion of a cell of one tissue lineage to entirely distinct lineage, with acquiring new markers and functions. This give rise to the concept of stem cell plasticity, allowing them to respond to a variety of micro environmental regenerative signals. The role of adult stem cells in tissue regeneration suggests lineage plasticity of adult stem cells populations. ${ }^{3}$ Asymmetric division explains the capability of stem cells to produce continuously differentiated cells and maintain their own population of undifferentiated cells. This raises the question regarding whether asymmetry of cell division is a prerequisite to explain the heterogeneity in the cellular fate of human SC. Self-organization changes cell-cell and cell-microenvironment interactions. Signaling environment induces developmental asymmetry that generates cell fate heterogeneity. The concept is consistent with the functional definition of tissue stem cells. ${ }^{4}$ Adult stem cells are defined by two cardinal properties: the ability

\section{To undergo self-renewal}

2. Differentiate into cell lineages present in a specific tissue Intrinsic and extrinsic cellular mechanisms regulate the balance of self-renewal and differentiation in all stem cells, which are asymmetrically localized at the interface between human stem cells (HSCs) and the niche.

\section{What is a niche of stem cells?}

The definition of a niche is the in vivo microenvironment that regulates stem cell survival, self-renewal and differentiation. ${ }^{5}$ Intrinsic and extrinsic cellular mechanisms regulate the balance of all stem cells. ${ }^{6}$ A stem cell niche is composed of micro environmental cells that maintain tissue homeostasis. From different studies it comes out that stem cell niches present an anatomical organization and co-ordinate

\author{
Volume 6 Issue 4 - 2017 \\ Michel Goldberg
Professeur Emerite, Biomédicale des Saints Pères, Université
Paris Descartes, France
}

Correspondence: Michel Goldberg, Professeur Emerite, Biomedicale des Saints Pares, Universite Paris Descartes, INSERM UMR-S I I 24, France, Email mgoldod@gmail.com

Received: February II, 2017| Published: February 23, 2017

cell functions in space and time. Both positive and negative signals are integrated. The Bmp/Wnt axis represents one such example. Intercellular signaling pathways are shared. The differentiation/ decision is influenced by a specialized microenvironment called stem cell niche. Interactions of the cleavage plane during stem cell mitosis control the balance between symmetric and asymmetric divisions. ${ }^{7}$ Niches provide an environment that sequesters stem cells from differentiation, apoptotic stimuli, and other stimuli that would challenge stem cell reserves. The niches control the number of stem cells in the body and protect it from excessive proliferation. Under particular conditions, stem cells can leave and return to their niches. Stem cells are retained in the niche by cell-to-cell interactions and adhesion to the extracellular matrix. Some data suggest that the secondary niches are localized around the blood vessels because they present a co-localization with pericytes. ${ }^{8}$ Inside the niche, stem cells are often quiescent. The adhesion molecules that appear to be important in niche function include $\mathrm{N}$ cadherin/ $\beta$-catenin, VCAM/ integrin, and osteopontin/ $\beta 1$ integrin (OPN $/ \beta$ integrin). ${ }^{9}$ They may play a role either for the attachment of stem cells to the niche or they are involved in the migration of stem cells. Two adherens junction molecules, $\mathrm{N}$-cadherin and $\beta$-catenin contribute to the formation of niches. A critical characteristic of the stem cell regulation is the microenvironment. It was proposed that there are not just several unique niche cells, but a wide variety of niche cells, which continually change phenotype to appropriately interact with the continuum of stem cell phenotypes. ${ }^{10} \mathrm{~A}$ stem cell gives rise through an asymmetric cell division to one stem daughter and another one that undergo differentiation. In contrast, highly regulative mechanisms in which a stem cell gives rise to daughter cells that have a finite probability of being either stem cells or committed progenitors. Most mammalian self-renewing tissues fall into this category. Both mechanisms involve multiple feedback controls and reciprocal intercellular interactions. The external signals that control stem cell microenvironment or niche involve a complex interplay of short- and long-range signals between stem cells, their differentiating daughters, and neighboring cells. Two families, the TGFb and Wnts show functional conservation between species and between tissues that self-renew through asymmetric divisions or population asymmetry. Integral membrane proteins, such as b-catenin, a structural component of adherens junctions, mediate cell-cell interactions. The receptor Notch and its ligand Delta are also implicated in this function. Integrins and extracellular matrix regulate the differentiation through MAP kinase signaling. ${ }^{11}$ Mesenchymal 
stem cells appear to be perivascular in human bone marrow and in dental pulp. ${ }^{8}$ Endothelial cells express the a-smooth muscle actin (von Willebrand factor-CD146) and a pericyte associated-antigen (3G5). They regulate the formation of mineralized matrices. The niche microenvironment is both proliferation- and differentiationinhibitory. Regulating stem cell self-renewal is an essential feature of the niche. The ability to divide symmetrically generates identical twins of most cells. In contrast, asymmetric cell divisions produce one committed progenitor daughter and one stem cell daughter. The balance between symmetric and asymmetric stem cells divisions maintains the proper stem cell number within the niche. It seems that stem cell niches provide a microenvironment protecting and perpetuating the self-renewing, maintain their stemness, determining how fast they divide and whether they divide symmetrically or asymmetrically. The niche 'adhesive' milieu allows retaining stem cell daughters, but expelling terminally differentiating daughters. ${ }^{12} \mathrm{~A}$ simple niche is a specific location in a tissue where stem cells can reside for an indefinite period of time and produce progeny cells while self-renewing. Complex niches are due to the concomitant presence of multiple stem cells that reside within a niche. Storage niches may contain quiescent stem cells. Daughter cells continue to divide and specialize after leaving the niche. They do not immediately lose the capacity to function as stem cells. Specific mechanisms are required to stabilize the early steps of differentiation, steps that can sometimes be reversed. ${ }^{13}$

\section{Signaling pathways of stem cells}

Signaling pathways have been identified, implicated in stem cells differentiation. They include the JAK/STAT pathway, NOTCH signaling, the MAP-kinase/ERK, the PI3K/AKT, the NFkB, the Wnt and the TGFb pathways. The JAK/STAT pathway proposes that cells communicate with each other through the secretion of cytokines, which are small $(8-30 \mathrm{kDa})$ soluble proteins. Roughly 20 cytokines have been identified. The JAK/STAT pathway plays important role in mediating cell fates such as apoptosis, differentiation and proliferation, in response to growth promoting factors and cytokines. In mouse embryonic stem cells (mESC), Notch signaling pathway consists of a protein that upon ligand binding undergoes proteolytic cleavage and release a transcription factor that shuttle to the nucleus. Notch inhibits the cellular differentiation within a tissue (lateral inhibition) or promotes adjacent cells to adopt the same cell fate (lateral induction). Notch signaling is not active in undifferentiated human ESC and do not requires the maintenance of pluripotency in human ESC. MAPK/ ERK pathways in stem cells are down regulated, less than four-fold. The results obtained suggest that MAPK/ERK signaling is active in undifferentiated hESC and down regulated upon differentiation. The PI3K/AKT signaling pathway maintains pluripotency in mouse ESC. Markers of pluripotency such as OCT4, NANOG and SOX2 decrease, indicating that the cells lost their Pluripotent capabilities and initiated differentiation. The NF-kappa B pathway is involved in proliferation, stress response, innate immunity and inflammation. NF-kB dimers are located in the cytoplasm and inactivated by their interaction with I-kB inhibitory proteins. The liberated NF-kB dimers are translocated to the nucleus and they activate transcription of target genes. Results lead to conclude that NF-kB is essential to maintain ESC pluripotency. The Wnt pathway has three branches: the $\mathrm{Ca}++$, the planar polarity, and the canonical branch, implicated in the maintenance of pluripotency ("stemness"). It consists of more than 30 extracellular Wnt-ligands, interacting with receptors of the frizzled family. Wnt ligand interacting with the receptor activates a protein called Dishelveld (Dsh), an inhibitor of the glycogenActivated kinase-3 (GSK-3). In the absence of Wnt signaling, GSK-3 phosphorylates and targets the b-catenin-Adenomatous Polyposis Coli (APC) for ubiquitinisation and proteolytic degradation. The inhibition of glycogen synthase kinase-3 (GSK-3) activity enhances HSC progenitor activity and maintains but does not expand the stem cell pool. Upon Wnt signaling, $\beta$-catenin is stabilized, accumulates in the cytoplasm and is translocated to the nucleus, where it interacts with DNA-binding proteins of the T-cell Factor / Lymphocyte Enhancer binding Factor (Tcf/Lef) family. In the presence of $\beta$-catenin, Tcf/Lef act as transcriptional activators of proliferation, stimulating genes such as c-myc and cyclin D1. In mammals, Wnt-signaling plays a crucial role in sustaining self-renewal in both embryonic and adult stem cells. The pharmacological inhibitor 6-bromoindirubin-3' oxime (BIO) blocks GSK-3. In the presence of BIO, b-catenin accumulates and translocates to the nucleus where it activates the transcription of genes involved in self-renewal. BIO maintains the undifferentiated state of human ES cells for several passages. BIO treated ESC retain the differentiation potential. TGF-b signaling and self-renewal in stem cells plays a crucial role in stem cell development, demonstrating the importance of this pathway in maintaining pluripotency and selfrenewal during embryogenesis in human embryonic stem cells. ${ }^{14}$

\section{Prospect: tissue repair and/or regeneration}

The identification of a rare population of adult stem cells in most tissues/organs in human has emerged as an attractive source of multipotent stem/progenitor cells for cell replacement-based therapies and tissue engineering in regenerative medicine. Diverse poorly differentiated adult stem/progenitor cell types have generally small size relative to the terminally differentiated cells. They express specific stemness markers, such as CD133, CD44, nestin and/or ABCG2 that have recently been identified in the most mammalian tissues/organs. ${ }^{15}$ The tissue regeneration mediated via adult stem/ progenitor cells is usually accompanied by environmental changes in the niche and orchestrated by several growth factor and cytokineinitiated cascades. Among them, epidermal growth factor (EGF)epidermal growth factor receptor (EGFR), sonic hedgehog (SHH)patched receptor $(\mathrm{PTCH}) / \mathrm{Gli}$, wingless ligand $(\mathrm{Wnt}) / \beta$-catenin, Notch, bone morphogenic proteins (BMPs), and stromal cell-derived factor-1 (SDF-1)-CXC chemokine receptor 4 (CXCR4) are tightly associated to this series of signaling pathways. The identification of the intrinsic and extrinsic factors that govern the decision between the self-renewal versus differentiation of tissue-resident adult stem cells, as well as the influence of the extracellular signals from their local microenvironment "niche" on their behavior in vivo, is also of interest for the design of new therapeutic strategies. The characterization of the biological properties of ex-vivo expanded adult stem/progenitor cells and their further differentiated progenies in diverse animal models in vivo after long-term treatment worth future investigations. Much research is focused on the identification of signals that maintain stem cells pluripotency or direct their differentiation. Gap junctions have been implicated in stem cells proliferation, differentiation and apoptosis. ${ }^{16}$ Gap junctions are hydrophilic channels, made by connexons, located in the membrane of adjacent cells. To date, 21 connexines have been identified in human and 20 in the mouse. Connexines consists of four transmembrane regions, two extracellular loops and three intracellular domains. The cytoplasmic C-terminal tail contains multiple serine and tyrosine residues, which allows phosphorylation, resulting a molecular diffusion through gap junctions. Somatic stem cells can undergo symmetric division to self- 
renew, or asymmetric division to generate a differentiated daughter cell. Mesenchymal Stem Cells (MSC) has been isolated from various tissues, including bone, umbilical cord blood and adipose, and will further differentiate into adipose tissue, cartilage and bone. Recently it was demonstrated in hESC that siRNA are able to move through gap junctions to affect gene expression of the neighboring cells.

\section{How many stem cells are found within a tissue? Isolation of mesenchymal stem cells and their use as therapeutic agent}

In marrow, the colony forming unit-fibroblastic (CFU-f) assay indicates $1 / 10.000$ for the newborn, $1 / 100.000$ at ten years, $1 / 250.000$ at 30 years, $1 / 400.000$ at 50 years, and $1 / 2.000 .000$ for an 80 years old human. ${ }^{17}$ Multilineage potential of adult mesenchymal stem cells indicates that the small percentage of stem cells is estimated at about 0.001 to $0.01 \% .^{18}$ MSCs represent 1 in 10,000 nucleated cells. Mesenchymal stem cells (MSCs) have been isolated from bone marrow, expanded in culture, and differentiated into several tissueforming cells such as bone, cartilage, fat, muscle, tendon, liver, kidney, heart and even brain cells. ${ }^{19}$ They may be used as therapeutic agents. This is especially obvious for dental tissues.

\section{Regeneration and repair of pulp and/or dental structures}

Tissues of the dental pulp and periodontal ligament were obtained from impacted third molars. The most accessible mesenchymal cells collectively termed dental stem cells (DSCs) include dental pulp stem cells (DPSCs), ${ }^{20}$ stem cells from exfoliated deciduous teeth, ${ }^{21,22}$ stem cells from the apical papilla (SCAP), ${ }^{22}$ periodontal ligament stem cells (PDLSCs), and dental follicle progenitor cells (DFPSc) ${ }^{24,27}$ Implanted adult human dental pulp stem cells induce endogenous axon guidance. ${ }^{28}$ Bone marrow MSCs (BMMSCs) have been described as colonyforming unit-fibroblasts (CFU-Fs). They give rise to various lineages of cells and commit osteogenic differentiation. They also differentiate into chondrogenic, adipogenic, myogenic, and neurogenic cells. TMSCs are found in many other tissues in the body, including umbilical cord blood, adipose tissue, adult muscle, and dental tissues. They are capable of differentiating into at least 3 cell lineages: osteogenic, chondrogenic, and adipogenic; and they can also differentiate into other lineages, such as odontogenic, when the cells are grown in a defined microenvironment in vitro. MSCs have immunosuppressive functions. The dental pulp is a potential source of MSCs instead of bone marrow (BM). Cells were equally positive for $\mathrm{SH}$, $\mathrm{SH}$, $\mathrm{SH} 4$, CD29 and CD166. These cells show osteogenic, chondrogenic and adipogenic lineages, but did not differentiate into chondrocytes. ${ }^{29}$ Digested in collagenase/dipase, single cell suspensions were obtained. Cultured in a-MEM supplemented with $20 \%$ fetal bovine serum; $2 \mathrm{mM}$ L-glutamine, $100 \mathrm{mM}$ L-ascorbate-2-phosphate, their phenotype was characterized. Co-transplantation with hydroxyapatite/tricalcium phosphate particles into immunocompromised mice for 8 weeks, mesenchymal stem cells were identified in adult dental pulp stem cells-DPSCs), human primary teeth (stem cells from exfoliated deciduous teeth, SHED), and periodontal ligament stem cells, PLSCs) for their capacity to generate clonogenic cell clusters in culture. ${ }^{30}$ The cells express a heterogeneous assortment of markers associated with the cells. The PDL includes Sharpey's fibers, embedded between the thin mineralized outer layer of cementum and the inner wall of the alveolar bone socket. Mesenchymal stem cell populations have been identified in adult bone marrow (BMSSCs) and dental pulp
(DPSCs). Isolated by immunoselection using the antibody STRO-1 that recognizes an antigen on perivascular cells, they were found to be positive for a-smooth muscle actin and CD146. Perivascular niche of post-natal SC were identified in human bone marrow and dental pulp. ${ }^{8}$ Bone marrow stromal stem cells (BMSSCs) and odontoblasts (DPSCs) form clonogenic cell clusters. It has been reported that two distinct primitive stem cell populations are intimately associated with the blood vessels of their respective tissues. Among the stem cells, some were identified as side population cells. They were isolated from porcine dental pulp tissue with self-renewal and multipotency for dentinogenesis, chondrogenesis, adipogenesis and neurogenesis. ${ }^{31}$ Based on the exclusion of DNA binding dye Hoechst 33342 by flow cytometry and compared with non-SP cells about $0.2 \%$ of the population was found to be side population. Expression of type II collagen and aggrecan confirmed chondrogenic conversion of SP cells. They express also the peroxisome proliferator-activated receptor and adaptator protein 2 showing adipogenic conversion. Neurofilaments and neuromodulin confirmed neurogenic conversion. BMP2 induce the differentiation of pulp SP into odontoblasts, stimulating the expression of Dspp and enammelysin. Isolation of concomittant multipotent and unipotent dental pulp progenitors allows demonstrating the coexistence of multipotential and restricted lineage progenitors in the mouse pulp. ${ }^{32}$ Mineralization potential of three cell lines was obtained from the mouse pulp (A4, H8 and C5 clones)? They display osteoblastic, osteodentin, chondrocytic and adipogenic differentiation, hence, phenotypically define distinct types of cell progenitors. Based on the sorting of subpopulations expressing different levels of cell surface molecules, they may be used for their repair potential in dental pulp and in other cranio-facial tissue lesions.

\section{Dental pulp stem cells (DPSC)}

\section{Immature dental pulp stem cells (IDPSC)}

Human embryonic stem cells express positive marker expression [Oct-4, Nanog, Rex-1 (transcription factor), and SSEA-3 and SSEA-4 (stage specific embryonic antigen), TRA-1-60 and TRA$1-81]$. The expression of these markers is maintained in subclones obtained from these cells. They can be induced to undergo uniform differentiation into smooth and skeletal muscles, neurons, cartilage and bone under chemically defined culture conditions. ${ }^{33}$ They may be characterized in vitro and in vivo. In vitro characterization of DPSCs: These cells possess dentinogenic potential, adipogenic and neurogenic differentiation capacities and they express the respective gene markers. In vivo characterization of DPSCs: Mixed with hydroxyapatite/tricalcium phosphate, they form dentin-pulp-like complex in immunocompromised mice. Expressing DSPP, a dentinlike structure is deposited on dentin surface. Adult post-natal pulp stem cells (DPSC) differentiation in vitro and tissue regeneration in vivo. Following transplantation, DPSCs are clonogenic cells, capable of self-renewal and Multilineage differentiation. DPSCs are able to regenerate a dentin-pulp-like complex composed of mineralized matrix, with tubules lined by odontoblasts and an arrangement similar to the dentin-pulp complex found in normal teeth. ${ }^{21-34}$ Pulp cells express bone markers such as bone sialoprotein, alkaline phosphatase, type I collagen and osteocalcin. Members of the TGFb super family and cytokines regulate their differentiation. They are similar to bone marrow stromal stem cells (BMSSCs) and their potential to develop into osteoblasts, chondrocytes, adipocytes, myelosupportive fibrous-stroma, muscle and neural tissues. They respond to specific environment signals, generate new stem cells and/or select a particular 
differentiation program. The phenotypical analysis evidenced that DPSCs were highly positive for CD29, CD44, CD90 and HLA I. They were negative for CD34, CD45, CD71, and HLA II. Postnatal dental pulp contains cells that are clonogenic, highly proliferative, and capable of regenerating a tissue. Hence, these properties define them as stem cells.

\section{Stem cells from human exfoliated deciduous teeth: SHED}

Exfoliated human deciduous tooth contains connective tissue, blood vessels and odontoblasts, acting as multipotent stem cells. The population of SHED was highly proliferative, clonogenic and capable of differentiating into a variety of cell types including neural cells, adipocytes, and odontoblasts. They were able to induce bone formation, generate dentin, and survive in mouse brain along with the expression of neural markers. SHEDs are derived from very accessible tissue resource but also they provide enough cells for potential clinical application. Miura et al..$^{22}$ concluded that exfoliated teeth may be a resource for stem-cell therapies including autologous stem cell transplantation and tissue engineering. In addition, SHED expressed STRO-1 and CD146, two early cell-surface markers for bone-derived MSCs. They expressed also Runx2, alkaline phosphatase (ALP), matrix extracellular phosphoglycoprotein (MEPE), bone sialoprotein (BSP), and DSPP. Implanted into immunocompromised mice with hydroxyapatite/tricalcium phosphate (HA/TCP), SHED differentiated into odontoblast-like cells forming dentin-like structures. The plasticity of postnatal stem cells taking origin from neural stem cells may contribute to blood and skeletal muscle, whereas bone marrow stem cells may contribute to muscle, liver, and neuronal tissue. Stem cells obtained from deciduous teeth may offer a unique stem-cell resource for potential clinical applications. ${ }^{22}$ Deciduous teeth contain a multipotent stem-cell population. They can be isolated and expanded ex vivo. They induce bone formation hosting osteogenic cells. SHED expressed neuronal and glial cell markers, probably related to the neural crest-cell origin of the dental pulp. SHED represents a population of postnatal stem cells capable of extensive proliferation and multipotential differentiation. Deciduous teeth therefore may be an ideal resource of stem cells to repair damaged tooth structures, induce bone regeneration, and possibly to treat neural tissue injury or degenerative diseases.

\section{Stem cells taking origin in the apical papilla (SCAP).}

These cells refer to the soft tissue located at the apex of developing permanent teeth. More apical to the epithelial diaphragm, they form an apical cell-rich zone underlying the apical papilla and the pulp. Root apical papilla of human teeth (SCAP) and periodontal ligament stem cells (PDLSCs) generates a root/periodontal complex resulting in normal tooth function. In vitro, the SCAPs are characterized by odontogenic differentiation. They express many surface markers including STRO-1, ALP, CD24, CD29, CD73, CD90, CD105, CD106, CD146, CD166, but were negative for CD34, CD45, CD18, and CD150. Stro-1 and CD146 have been identified as early specific marker for SCAP, not detectable in other mesenchymal stem cells including DPSCs and BMMSCs. Hence, SCAP derived from a developing tissue represent a population of early progenitors that have advantages for use in tissue regeneration. ${ }^{23}$ SCAP expresses also low levels of DSP, matrix extracellular phosphoglycoprotein (MEPE), transforming growth factor $\mathrm{b}$ receptor II (TGFbRII), FGFR3, VGE receptor 1, and melanoma-associated glycoprotein.
The expression of CD24 by SCAP is down regulated in response to osteogenic stimulation with SCAP, and demonstrates the capacity to undergo adipogenic differentiation following induction in vitro. In vitro cultured SCAP show positive staining for several neuronal markers, including glutamic acid decarboxylase, neuronal nuclear antigen, neurofilament, neural-specific enolase and glial markers. In continued root formation, SCAP characteristics are similar to those of DPSCs, and they may be the source of replacement odontoblasts. ${ }^{35}$ They are responsible for the formation of root dentin. In a pilot study, the role of apical papilla in root formation containing apical papilla is impaired after surgical removal, whereas normal growth and development is maintained despite the pulp remains intact. Apexogenesis and apexification are resulting from a common origin of dental stem cells. They make up to only $1-4 \%$ of cells, which may include progenitor cells. The stem cell population in the pulp is very small, approximately $1 \%$ of the total cells. ${ }^{36}$ Gene profile analysis have identified perivascular cell markers, CD146/MUC18, 3G5, CD44, VCAM-1, alkaline phosphatase and a-smooth muscle actin in differing proportions on STRO-1 positive cells from dental tissues. Pulp cells are able to proliferate and differentiate into odontoblast-like cells with processes extending into the dentinal tubules. Regeneration of tissue into the apex of an immature permanent tooth may come from stem cells already residing in vital pulp tissue, the apical papilla, PDL or alveolar bone; alternatively, stem cells and growth factors seeded on scaffolds may be used to regenerate tissue in vitro or in vivo.$^{37}$ It was concluded that apical papilla harbors multipotent MSCs that express various MSC markers. They are capable of forming odontoblast-like cells and produce dentin in vivo and are likely to be the cell source of primary odontoblasts for the root dentin formation. ${ }^{38}$

\section{Periodontal ligament stem cells (PDLSCs)}

The PDL contains cells populations different from the entire cementforming cells (cementoblasts) and bone-forming cells (osteoblasts). These cells maintain tissue homeostasis and regeneration. Digestion of the PDL suggests that the tissue contains progenitor cells. Enzyme digestion release a population of clonogenic ${ }^{39}$ cells PDLSCs are clonogenic, highly proliferative cells and capable of regenerating cementum/PDL-like tissues. They express the mesenchymal stemcell markers STRO-1 and CD146/MUC18. Under defined culture conditions PDLSCs differentiate into cementoblast-like cells, adipocytes and collagen-forming cells. They express cementoblastic/ osteoblastic markers including alkaline phosphatase, MEPE, bone sialoprotein, osteocalcin and TGFb receptor type I. After three weeks culture with an adipogenic cocktail, PDLSCs differentiate into Oil-redO-positive adipocytes [40]. Transplanted into immunocompromised rodents, PDLSCs generate a cementum/PDL-like structure and contribute to periodontal tissue repair. ${ }^{40}$

\section{Dental follicle precursor cells (DFPCs)}

Dental follicle is an ectomesenchymal tissue surrounding the enamel organ and dental papilla of the tooth germ prior to eruption. The tissue contains progenitor cells forming the periodontium (cementum, PDL and alveolar bone). Clonogenic colonies are released from the tissue following enzymatic digestion. ${ }^{24-41}$ In vitro, markers such as Notch-1 and nestin are expressed. Stem cells were identified in the dental follicle by Hoechst staining, alkaline phosphatase staining and expression of side-population markers. ${ }^{42}$ Nestin, Notch-1, type I collagen, bone sialoprotein (BSP), osteocalcin and fibroblast growth factor receptor (FGFR) 1-IIIC are expressed. In addition STRO-1 
and BMP receptors (BMPR) are expressed in vivo in dental follicles. The cementum attachment protein and cementum protein-23 were detected in EMD (Enamel matrix derivatives- Emdogain)-stimulated whole dental follicle and in cultured DFPCs stimulated with EMD or BMP-2 and BMP-7. In vivo, transplants expressed human-specific transcripts for BSN, OCN and type I collagen. Gene's transcription was increased by more than 100 times for BSP and OCN, and decreased for Type I collagen after implantation in immunocompromised mice. However, no dentin, cementum or bone formation was observed in the transplant.

iPS: Induced Pluripotent Stem cells (iPS) may be generated. Many types of cells have been successfully reprogramed into iPS cells in the mouse system. However, reprogramming human cells seems to be more difficult. Stem/progenitor cells from discarded dental tissues can be reprogrammed into iPS cells. ${ }^{43}$ Human dermal fibroblasts were the first type of cells being reprogrammed into iPS cells. The 4 factors Lin28/Nanog/Oct4/Sox2 or c-Myc/Klf4/Oct4/ Sox2 carried by viral vectors were used to reprogram three different dental stem/progenitor cells: 1) stem cells from exfoliated deciduous teeth (SHED), 2) stem cells from apical papilla (SCAP), and 3) dental pulp stem cells (DPSCs). They expressed the cell gene markers SSEA-4, TRA-160, TRA-1-80, TRA-2-49, Nanog, Oct4, and Sox2. They formed embryoid bodies in vitro and teratomas in vivo containing tissues of all three germ layers. Further studies represent another step toward establishing protocols to generate iPS cells using an easily accessible alternative cell source. Further investigations are critical

a. Generate dental stem cell-derived iPS.

b. To characterize iPS cells from other cell sources.

c. Establish reliable protocols to guide iPS cells toward differentiated cells for specific tissue regeneration.

d. Determine the genomic stability of iPS cells in long-term culture. ${ }^{43}$

\section{Future prospects}

Effective cellular-based therapies for regenerative medicine have been elaborated.

a. Which mechanism of self-renewal will regulate adult stem cell growth to generate a sufficient number of cells needed for different applications.

b. During differentiation, the production of extracellular material and its maturation involve a cascade of signals.

c. Understanding the interaction between stem cells and the immune system allows clinical applications. However, the question arises on the need to determine if allogenic dental MSC promote short- and long-term immunorejection.

d. Preventing ex-vivo expanded MSCs for transformation, allows adipose-derived MSCs from their lost genetic stability over time.

\section{Funding}

None.

\section{Acknowledgements}

None.

\section{Conflicts of interest}

The authors declare that there is no conflict of interest.

\section{References}

1. Körbling M, Estrov Z. Adult stem cells for tissue repair- a new therapeutic concept?. New England Journal Medicine. 2003;349: 570-582.

2. Da Silva Meirelles L, Chagastelles PC, Nardi NB. Mesenchymal stem cells reside in virtually all post-natal organs and tissues. J Cell Sci. 2006;119(pt 11):2204-2213.

3. Wagers AJ, Weissman IL. Plasticity of adult stem cells. Cell. 2004;116(5):639-648

4. Roeder I, Lorenz E. Asymmetry of Stem Cell Fate and the Potential Impact of the Niche. Stem Cell Rev. 2006;2(3):171- 180.

5. Discher DE, Mooney DJ, Zandstra PW. Growth factors, matrices, and forces combine and control stem cells. Science. 2010;324(5935):16731677.

6. Moore KA, Lemischka IR. Stem cells and their niches. Science. 2006;311(5769):1880-1885.

7. Mitsiadis T, Barrandon O, Rochat A, et al. Stem cell niches in mammals. Exp Cell Res. 2007;313(16):3377-3385

8. Shi S, Gronthos S. Perivascular niche of postnatal mesenchymal stem cells in human bone marrow and dental pulp. J Bone Miner Res. 2003;18(4):696-704.

9. Yin T, Li L. The stem cell niches in bone. $J$ Clinical Investigation 2006;116(5):1195-1201.

10. Quesenberry PJ, Aliotta JM. The paradoxical dynamism of marrow stem cells: considerations of stem cells, niches, and microvesicles. Stem Cell Rev. 2008;4(3):137-147.

11. Watt FM, Hogan BLM. Out of Eden: stem cells and their niches. Science. 2000;287(5457):1427-1430.

12. Fuchs E, Turnbar T, Guasch G. Socializing with the neighbors: stem cells and their niche. Cell. 2004;116(6):769-778.

13. Ohlstein B, Kai T, Decotto E, et al. The stem cell niche: theme and variations. Current Opinion in Cell Biology. 2004;16(6):693-699.

14. Dreesen O, Brivanlou AH. Signaling pathways in cancer and embryonic stem cells. Stem Cell Rev. 2007;3(1):7-17.

15. Mimeault M, Batra SK (2008) Recent progress on tissue-resident adult stem cell biology and their therapeutic implications. Stem Cell Rev 4(1): $27-49$.

16. Wong RCB, Pera M, Pébay A. Role of gap junctions in embryonic and somatic stem cells. Stem Cell Rev. 2008; 4(4):283-292.

17. Caplan AI. Adult Mesenchymal Stem Cells for tissue engineering versus regenerative medicine. J Cell Physiol. 2007;213(2): 341-347.

18. Pittenger MF, Mackay AM, Beck SC, et al. Multilineage potential of adult human mesenchymal stem cells. Science. 284(5411): 143-147.

19. Alhadlaq A, Mao JJ. Mesenchymal Stem Cells: isolation and therapeutics. Stem cells Dev. 2004;13(4):436-448.

20. Gronthos S, Mankani M, Brahim J, et al. Postnatal human dental pulp stem cells (DPSCs) in vitro and in vivo. Proc Natl Acad Sci USA 2000;97(25):13625-13630

21. Seo BM, Sonoyama W, Yamaza T, et al. SHED repair critical-size calvarial defects in mice. Oral Dis. 2008;14(5):428-434. 
22. Miura M, Gronthos S, Zhao M, et al. SHED: Stem cells from human exfoliated deciduous teeth. Proc Natl Acad Sci USA. 2003; 100(10): $5807-5812$.

23. Sonoyama W, Liu Y, Fang D, et al. Mesenchymal stem cell-mediated functional tooth regeneration in swine. Plos one. 2006; 1(1):e79.

24. Morsczeck C, Gotz W, Schierholz J, et al. Isolation of precursor cells (PCs) from human dental follicle of wisdom teeth. Matrix Biol. 2005;24(2):155-165.

25. Huang GT. A paradigm shift in endodontic management of immature teeth: conservation of stem cells for regeneration. J Dentistry. 2008;36(6):379_ 386.

26. Huang GT, Gronthos S, Shi S. Mesenchymal stem cells derived from dental tissues vs. those from other sources: their biology and role in regenerative medicine. J Dent Res. 2009;88(9):792-806.

27. Sedgley CM, Botero TM. Dental stem cells and their sources. Dent Clin N Am. 2012;56(3):549-561.

28. Arthur A, Shi S, Zannettino A, et al. Implanted adult human dental pulp stem cells induce endogenous axon guidance. Stem cells. 2009;27(9):2229-2237.

29. Pierdomenico L, Bonsi L, Calvitti M, et al. Multipotent mesenchyma stem cells with immunosuppressive activity can be easily isolated from dental pulp. Transplantation. 2005;80(6):836-842.

30. Shi S, Bartold PM, Miura M, et al. The efficacity of mesenchymal stem cells to regenerate and repair dental structures. Orthod Craniofac Res. 2005;8(3):191-199.

31. Iohara $\mathrm{K}$, Zheng L, Ito $\mathrm{M}$, et al. Side population cells isolated from porcine dental pulp tissue with self-renewal and multipotency for dentinogenesis, chondrogenesis, adipogenesis, and neurogenesis. Stem cells. 2006;24(11):2493-2503.

32. Lacerda Pinheiro S, Dimitrova Nakov S, Harichane Y, et al. Concomitant multipotent and unipotent dental pulp progenitors and their respective contribution to mineralized tissue formation?. Eur Cells \& Materials. 2012;23:371-386.
33. Kerkis I, Kerkis A, Dozortzev D, Chopin Stuart PG, et al. Isolation and characterization of a population of immature dental pulp stem cells expressing Oct-4 and other embryonic stem cells markers. Cell Tissues Organs. 2006;184(3-4):105-116.

34. Gronthos S, Brahim J, Li W, et al. Stem cell properties of human dental pulp stem cells. J Dent Res. 2002;81(8):531-535.

35. Sonoyama W, Liu Y, Yamaza T, et al. Characterization of the apical papilla and its residing stem cells from human immature permanent teeth: a pilot study. J Endod. 2008;34(2):166-171.

36. Sloan AJ, Waddington RJ. Dental pulp stem cells: what, where, how?. Int J Paediatr Dent. 2009;19(1):61-70.

37. Friedlander LT, Cullinan MP, Love RM. Dental stem cells and their potential role in apexogenesis and apexification. Int Endod J. 2009;42:955-962.

38. Seo BM, Sonoyama W, Yamaza T, et al. SHED repair critical-size calvarial defects in mice. Oral Dis. 2008;14(5):428-434.

39. Seo B, Miura M, Gronthos S, et al. Investigation of multipotent postnatal stem cells from human periodontal ligament. Lancet. 2004;364(9429):149-155.

40. Mao JJ, Giannobile WV, Helms JA, et al. Craniofacial tissue engineering by stem cells. J Dent Res. 2006;85(11):966-979.

41. Honda MJ, Imaizumi M, Tssuchiya S, et al. Dental follicle stem cells and tissue engineering. J Oral Sci. 2010;52(4):541-552.

42. Yao S, Pan V, Prpic V, Wise GE. Differentiation of stem cells in the dental follicle. J Dent Res. 2008;87(8):767-771.

43. Yan X, Qin H, Qu C, et al. iPS cells reprogrammed from mesenchymallike stem/progenitor cells of dental tissue origin. Stem Cells Dev. 2010;19(4):469-480. 